\title{
Divergence-free vector-field and reduction
}

\author{
Debin Huang ${ }^{\mathrm{a}}$, Xiaohua Zhao ${ }^{\mathrm{b}}$, Zengrong Liu ${ }^{\mathrm{a}}$ \\ ${ }^{a}$ LNM, Institute of Mechanics, Academy of China and Mathematical Department of Shanghai University, Shanghai 201800, China \\ ' Mathematical Department of Yunnan University. Kuiming 650091, China
}

Received 6 January 1998; revised manuscript received 18 April 1998; accepted 21 April 1998

Communicated by C.R. Doering

\begin{abstract}
By the Lie symmetry group, the reduction for divergence-free vector-fields (DFVs) is studied, and the following results are found. A $n$-dimensional DFV can be locally reduced to a $(n-1)$-dimensional DFV if it admits a one-parameter symmetry group that is spatial and divergenceless. More generally, a $n$-dimensional DFV admitting a $r$-parameter, spatial, divergenceless Abelian (commutable) symmetry group can be locally reduced to a $(n-r)$-dimensional DFV. (C) 1998 Elsevier Science B.V.
\end{abstract}

\section{Introduction}

Recently, there has been increasing interest in the DFVs that preserve some differential form, see, e.g., Refs. [1,2].

On the basis of the symplectic reduction preserving the Hamiltonian structure [3], we obtain the reduction preserving the divergenceless nature by the Lie symmetry group, i.e. a $n$-dimensional DFV can be locally reduced to a $(n-1)$-dimensional DFV when it admits a one-parameter, spatial, divergenceless symmetry group. More generally, a $n$-dimensional DFV admitting a $r$-parameter, spatial, divergenceless Abelian (commutable) symmetry group can be locally reduccd to a $(n-r)$-dimensional DFV. This result is a generalization in the spirit of the symplectic reduction for Hamiltonian systems [3].

Note that our results contain those in Ref. [4,5], and also coincide with the conclusion in Ref. [6]. But our proof is more simple than the one in Ref. [6], and the conditions demanded in this paper are weaker than those in Ref. [6]. More important is that such a reduction simplifies the study of some nonlinear dynamical systems, see, e.g., Refs. [5,7]. From a theoretical point of view, however, the main shortcoming is the introducing of coordinate systems in our proof because we hope to obtain concrete reduced systems in order to study their dynamics in an application.

\section{Divergence-free vector-fields and symmetry group}

Definition 1 . Let $M$ be a $n$-dimensional manifold, and $\Omega$ be a $n$-form on $M$. For any vector-field $F$ on $M$, if $L_{F} \Omega=0$, where $L_{F}$ is the Lie derivative along the flows of $F$, the vector-field is said to preserve the $n$-form $\Omega$.

With the local coordinates $\left(x^{1}, \ldots, x^{n}\right)$ on $M$, consider $\Omega$ as the canonical $n$-form $\Omega=\mathrm{d} x^{1} \wedge \ldots \wedge \mathrm{d} x^{n}$. Then we have $L_{F} \Omega=\operatorname{div}(F) \Omega$, where 
$\operatorname{div} F=\sum_{i=1}^{n} \frac{\partial f_{i}\left(x^{1}, \ldots, x^{n}\right)}{\partial x^{i}}$

and $F$ is denoted by

$$
\begin{aligned}
\frac{\mathrm{d} x^{i}}{\mathrm{~d} t} & =f_{i}\left(x^{1}, \ldots, x^{n}, t\right), \quad\left(x^{1}, \ldots, x^{n}\right) \in M, \\
t & \in R, \quad i=1, \ldots, n .
\end{aligned}
$$

Thus saying the vector field $F$ preserves the $n$-form is equivalent to saying $F$ is a divergence-free vectorfield (DFV). We prefer the latter in this paper.

Definition 2. Let $G$ be a Lie group acting on $M \times R$. If $G$ satisfies the following conditions: (i) $G$ is a symmetry group of the system (2.1), (ii) the infinitesimal generator $V$ of $G$ is

$V=\sum_{i=1}^{n} \xi^{i}\left(x^{1}, \ldots, x^{n}\right) \frac{\partial}{\partial x^{i}}$,

then $G$ is called a spatial symmetry group of the system (2.1). Furthermore, if $V$ satisfies the condition

$\sum_{i=1}^{n} \frac{\partial \xi^{i}\left(x^{i}, \ldots, x^{n}\right)}{\partial x^{i}}=0$,

then $G$ is referred to as a spatial, divergenceless symmetry group of (2.1).

Theorem 3 . The Lie group $G$ generated by

$V=\sum_{i=1}^{n} \xi^{i}\left(x^{1}, \ldots, x^{n}\right) \frac{\partial}{\partial x^{i}}$

(hereafter denoted by $\left.V=\left(\xi^{1}, \ldots, \xi^{n}\right)\right)$ is a spatial symmetry group of the system (2.1) if and only if $[F, V]=0$, where $F=\left(f_{1}, \ldots, f_{n}\right) .[F, V]$ denotes the Lie bracket of $F$ and $V$, defined in coordinates by

$[F, V]_{i}=\sum_{j-1}^{n}\left(f_{j} \frac{\partial \xi^{i}}{\partial x^{j}}-\xi^{j} \frac{\partial f_{i}}{\partial x^{j}}\right), \quad i=1, \ldots, n$.

Proof. This is an easy calculation, applying Theorems 2.36 and 2.71 in Ref. [8].

Theorem 4. Let the system (2.1) admit a oneparameter symmetry group $G$ whose infinitesimal generator is $V$, then there exists a local transformation of variables defined near the point $(x, t)$ at which $\left.V\right|_{(x, t)} \neq 0$, given by

$$
\begin{aligned}
x^{i} & =\eta_{i}\left(y^{1}, \ldots, y^{n}, s\right), \quad t=\varphi\left(y^{1}, \ldots, y^{n}, s\right), \\
& i=1, \ldots, n,
\end{aligned}
$$

such that the system (2.1) becomes

$\frac{\mathrm{d} y^{i}}{\mathrm{~d} s}=g_{i}\left(y^{1}, \ldots, y^{n-1}, s\right), \quad i=1, \ldots, n$,

where $y^{1}, \ldots, y^{n-1}$ and $s$ are a complete set of functional independent invariants of $V$, i.e.

$V\left(y^{i}\right)=0(i=1, \ldots, n-1)$,

$V(s)=0, \quad V(y)^{n}=1$.

Proof. We refer to Theorem 2.66 in Ref. [8].

Remark. Because the right-hand side of the system (2.3) is independent of $y^{n}$, the component $y^{n}$ can be got by quadrature. Thus we call usually the first $n-1$ equations of the system (2.3) a reduced system of (2.1) under $G$.

In particular, when $G$ is a spatial symmetry group, we get the following corollary.

Corollary 5. Suppose $G$ in Theorem 4 is a spatial symmetry group, then for the transformation (2.2) we can take $s=t$ and $\eta_{i}(i=1, \ldots, n)$ are independent of $s$, i.e. $y^{i}(i=1, \ldots, n)$ are independent of time.

Proof. Since $G$ is a spatial symmetry group, the function $t$ is just an invariant of $G$, and we can take $s=t$. Further, since

$V(y)=\sum_{i=1}^{n} \xi^{i} \frac{\partial y}{\partial x^{i}}$

where $\xi^{i}(i=1, \ldots, n)$ are independent of $t$, the solutions to the equations $V(y)=0$ and 1 are all independent of $t$. This completes the proof.

\section{Main results and proof}

Lemma 6. Suppose there exists a diffeomorphism $\varphi$, 
$x^{i}=\varphi_{i}\left(y^{1}, \ldots, y^{n}\right), \quad i=1, \ldots, n$,

under which the system (2.1) becomes

$\frac{\mathrm{d} y^{i}}{\mathrm{~d} t}=g_{i}\left(y^{1}, \ldots, y^{n}, t\right), \quad i=1, \ldots, n$.

Then the following relation is valid,

$\sum_{i=1}^{n} \frac{\partial f_{i}}{\partial x^{i}}=\frac{1}{|J|} \sum_{i=1}^{n} \frac{\partial\left(|J| g_{i}\right)}{\partial y^{i}}$,

where $J$ is the Jacobian matrix of the transformation $\varphi,|J|$ is its determinant.

Proof. Using the properties of the derivative and determinant, after a straightforward and lengthy calculation, one can prove the result.

Theorem 7. Let the vector-field (2.1) be divergencefree and admit a one-parameter, spatial, divergenceless symmetry group $G$. Then there exists a local transformation of variables such that the reduced vector-field of $(2.1)$ is a $(n-1)$-dimensional DFV.

Proof. Let the infinitesimal generator of $G$ be $V=$ $\left(\xi^{1}, \ldots, \xi^{n}\right)$. Applying Theorem 4 and Corollary 5 , there exists a transformation of coordinates as (3.1) under which $(2.1)$ becomes

$\frac{\mathrm{d} y^{i}}{\mathrm{~d} t}=k_{i}\left(y^{\mathrm{t}}, \ldots, y^{n-1}, t\right), \quad i=1, \ldots, n$.

According to Lemma 6, we get

$\sum_{i=1}^{n} \frac{\partial\left(|J| k_{i}\right)}{\partial y^{i}}=0$

Further, applying (2.4) in Theorem 4, we find the vector-field $V$ will have the form $(0, \ldots, 0,1)$ under $\varphi$. Using Lemma 6 again, we get

$$
\begin{aligned}
0 & =\sum_{i=1}^{n} \frac{\partial \xi^{i}}{\partial x^{i}}=\frac{1}{|J|}\left(0+\cdots+0+\frac{\partial|J|}{\partial y^{n}}\right) \\
& \Rightarrow \frac{\partial|J|}{\partial y^{n}}=0 .
\end{aligned}
$$

Thus $|J|$ is independent of $y^{n}$, and (3.5) becomes

$$
\sum_{i=1}^{n-1} \frac{\partial\left(|J| k_{i}\right)}{\partial y^{i}}=0 \text {. }
$$

Now consider the reduced system of (2.1), i.e. the first $n-1$ equations of the system (3.4),

$$
\begin{aligned}
\frac{\mathrm{d} y^{i}}{\mathrm{~d} t} & =k_{i}\left(y^{1}, \ldots, y^{n-1}, t,\right), \\
i & =1, \ldots, n-1 .
\end{aligned}
$$

Take a transformation $\Gamma$,

$$
\begin{aligned}
& z^{1}=\int|J| \mathrm{d} y^{1}, \\
& z^{2}=y^{2}, \quad \ldots, \quad z^{n-1}=y^{n-1},
\end{aligned}
$$

under which (3.7) becomes

$$
\begin{aligned}
\frac{\mathrm{d} z^{i}}{\mathrm{~d} t} & =g_{i}\left(z^{1}, \ldots, z^{n-1}, t\right), \\
i & =1, \ldots, n-1 .
\end{aligned}
$$

Obviously, the inverse $\Gamma^{-1}$ exists. In other words, (3.9) becomes (3.7) under $\Gamma^{-1}$. We calculate the Jacobian matrix $\mathrm{D} z / \mathrm{Dy}\left(z=\left(z^{1}, \ldots, z^{n}\right), y=\right.$ $\left.\left(y^{1}, \ldots, y^{n}\right)\right)$ of $\Gamma^{-1}$,

$\frac{\mathrm{D} z}{\mathrm{D} y}=\left[\begin{array}{cccc}|J| \frac{\partial}{\partial y^{2}} \int|J| \mathrm{d} y^{1} & \cdots & \frac{\partial}{\partial y^{n-1}} \int|J| \mathrm{d} y^{1} \\ 0 & 1 & \cdots & 0 \\ \vdots & & & \vdots \\ 0 & 0 & \cdots & 1\end{array}\right]$.

Thus applying Lemma 6 and (3.6), we have

$$
\begin{aligned}
& \sum_{i=1}^{n-1} \frac{\partial g_{i}}{\partial z^{i}}=\frac{1}{|D z / D y|} \sum_{i=1}^{n-1} \frac{\partial\left(|D z / D y| k_{i}\right)}{\partial y^{i}} \\
& =\frac{1}{|J|} \sum_{i=1}^{n-1} \frac{\partial\left(|J| k_{i}\right)}{\partial y^{i}}=0 .
\end{aligned}
$$

To sum up, for the vector-field (2.1) satisfying the conditions in Theorem 7 , there exists a transformation of coordinates: $x \rightarrow z$, under which (2.1) becomes

$\frac{\mathrm{d} z^{i}}{\mathrm{~d} t}=g_{i}\left(z^{1}, \ldots, z^{n-1}, t\right), \quad i=1, \ldots, n-1$,

$\sum_{i=1}^{n-1} \frac{\partial g_{i}}{\partial z^{i}}=0, \quad i=1, \ldots, n-1$, 
$\frac{\mathrm{d} z^{n}}{\mathrm{~d} t}=g_{n}\left(z^{1}, \ldots, z^{n-1}, t\right), \quad z^{n}=y^{n}$.

The theorem is proved.

Remark. From the above proof, one may find the transformation taking (2.1) to (3.11) does not depend on the vector-field (2.1), but only depends on the symmetry group $G$.

When $n=3$, from Theorem 7 , we get directly the main results in Refs. [4,5].

Corollary 8 . Let the following three-dimensional system be divergence-free,

$\frac{\mathrm{d} x^{i}}{\mathrm{~d} t}=f_{i}\left(x^{1}, x^{2}, x^{3}, t\right), \quad i=1,2,3$,

and admit a one-parameter, spatial, divergenceless symmetry group $G$. Then there exists a local transformation of coordinates,

$x^{i}=\varphi_{i}\left(z^{1}, \ldots, z^{\prime \prime}\right), \quad i=1,2,3$,

such that in new variables the system (3.12) becomes

$\frac{\mathrm{d} z^{1}}{\mathrm{~d} t}=\frac{\partial H\left(z^{1}, z^{2}, t\right)}{\partial z^{2}}$,

$\frac{\mathrm{d} z^{2}}{\mathrm{~d} t}=-\frac{\partial H\left(z^{1}, z^{2}, t\right)}{\partial z^{1}}$,

$\frac{\mathrm{d} z^{3}}{\mathrm{~d} t}=k_{3}\left(z^{1}, z^{2}, t\right)$.

where $H\left(z^{1}, z^{2}, t\right)$ is a certain function.

Proof. From Theorem 7, the two-dimensional reduced system of (3.12) must he a two-dimensional DFV, henceforth it can be expressed as a Hamiltonian form. So the system (3.12) can be taken to the form (3.13).

Now consider the case of a multi-parameter group.

Theorem 9. Let

$\frac{d x^{i}}{d t}=f_{i}\left(x^{1}, \ldots, x^{n}, t\right), \quad i=1, \ldots, n$,

be a $n$-dimensional DFV, and admit a two-parameter symmetry group $G$ that satisfies the following conditions: (i) $V_{1}$ and $V_{2}$ are divergenceless,

(ii) $\left[V_{1}, V_{2}\right]=0$, i.e. $G$ is an Abelian group,

where $V_{1}$ and $V_{2}$ are two independent infinitesimal generators of $G$. Then (3.14) can be locally reduced to a $(n-2)$-dimensional DFV.

Proof. We first reduce (3.14) by $V_{1}$ as the proof of Theorem 7. For simplicity, we still suppose the system (3.14) has the same reduced system as (3.7),

$\frac{\mathrm{d} y^{i}}{\mathrm{~d} t}=k_{i}\left(y^{1}, \ldots, y^{n-1}, t\right), \quad i=1, \ldots, n-1$.

under the same transformation as $\varphi$,

$x^{i}=\varphi_{i}\left(y^{1}, \ldots, y^{n}\right), \quad i=1, \ldots, n$.

According to the theory of Lie group, the quotient group $G / G_{1}$ is a symmetry group of (3.7), where $G_{1}$ is a Lie group generated by $V_{1}$, and $G / G_{1}$ is just a one-parameter group whose infinitesimal generator is the reduced vector-field of $V_{2}$ (written as $V_{3}$ ) under the complete set of invariants of $V_{1}$. Let

$V_{2}=\eta^{1} \frac{\partial}{\partial x^{1}}+\cdots+\eta^{n} \frac{\partial}{\partial x^{n}} \equiv\left(\eta^{1}, \ldots, \eta^{n}\right)$.

We work out $V_{3}$ below.

From Theorem 4, the complete set of invariants of $V_{1}$ is $\left\{t, y^{1}, \ldots, y^{n-1}\right\}$, then we get new coordinates $\left\{t, y^{1}, \ldots, y^{n-1}, y^{n}\right\}$ by supplementing a variable $y^{n}$ that is from $\varphi$. Under the new coordinates, let $V_{2}$ be taken correspondingly to

$$
\begin{aligned}
& \tilde{\eta}\left(y^{1}, \ldots, y^{n-1}\right) \frac{\partial}{\partial y^{1}}+\cdots \\
& \quad+\tilde{\eta}^{n-1}\left(y^{1}, \ldots, y^{n-1}\right) \frac{\partial}{\partial y^{n-1}} \\
& \quad+\tilde{\eta}^{n}\left(y^{1}, \ldots, y^{n-1}, y^{n}\right) \frac{\partial}{\partial y^{n}} .
\end{aligned}
$$

Thus

$$
\begin{aligned}
V_{3} & =\tilde{\eta}^{1}\left(y^{1}, \ldots, y^{n-1}\right) \frac{\partial}{\partial y^{1}}+\cdots \\
& +\tilde{\eta}^{n-1}\left(y^{1}, \ldots, y^{n-1}\right) \frac{\partial}{\partial y^{n-1}} .
\end{aligned}
$$

On the other hand, since $\left[V_{1}, V_{2}\right]=0$, from Theorem 3 , the autonomous system associated with $V_{2}$,

$$
\frac{\mathrm{d} x^{i}}{\mathrm{~d} t}=\eta^{i}\left(x^{i}, \ldots, x^{n}\right), \quad i=1, \ldots, n,
$$


admits a one-parameter symmetry group $G_{1}$ generated by $V_{1}$. The transformation in Theorem 7 is independent of the vector-fields, so we can transform (3.15) with the above transformation $\varphi$ similarly, and let the resulting system be

$$
\frac{\mathrm{d} y^{i}}{\mathrm{~d} t}=h^{i}\left(y^{1}, \ldots, y^{n-1}\right), \quad i=1, \ldots, n .
$$

From Ref. [1], we have

$h^{i}=\tilde{\eta}^{i}, \quad i=1, \ldots, n$.

Thus the system (3.7) admits a one-parameter symmetry group generated by $V_{3}=\left(h^{1}, \ldots, h^{n-1}\right)$, i.e. $\left[V_{3}, K\right]=0, K=\left(k_{1}, \ldots, k_{n-1}\right)$.

Further, for (3.7) and (3.16), we take the second transformation similar to $\Gamma$ in the proof of Theorem 7 , and we still suppose (3.7) is taken to (3.9) under $\Gamma$. On the other hand, from Theorem 7, the first $n-1$ equations of (3.16) are taken to an autonomous ( $n-$ 1)-dimensional DFV (written as $V_{3}^{*}$ ).

From $\left[V_{3}, K\right]=0$, one gets easily $\left[V_{3}^{*}, g\right]=0$, where $g=\left(g_{1}, \ldots, g_{n-1}\right)$ defined in (3.9) (this is because the definition of the Lie bracket is independent of the choice of coordinates). Thus the system (3.9) admits a spatial, divergenceless symmetry group generated by $V_{3}^{*}$, then applying Theorem 7 again, the vector-field ( 3.14$)$ is reduced to a $(n-2)$-dimensional DFV. This theorem is proved.

More generally, one can obtain the following result from the above proof.

Theorem 10. If a $n$-dimensional DFV admits a $r$-parameter spatial, divergenceless Abelian (commutable) symmetry group, then it can be locally reduced to a $(n-r)$-dimensional DFV.

In Theorem 10, when $r=n-2$, from Corollary 8 , the reduced vector-field is an integrable Hamiltonian system with one degree of freedom, so the original system can be integrated by quadrature. This result coincides with the conclusion in Ref. [6], without the condition on rank as in Ref. [6].

\section{An example}

Consider the modified model from Ref. [9], $\dot{u}=-v w+b v z, \quad \dot{v}=u w-b u z$,

$\dot{w}=-\frac{1}{2}\left(u^{2}+v^{2}\right), \quad \dot{x}=-z$,

$\dot{z}=x+\frac{1}{2} b\left(u^{2}+v^{2}\right)$.

It is shown easily that (4.1) admits a one-parameter symmetry group whose infinitesimal generator is

$\xi=-v \frac{\partial}{\partial u}+u \frac{\partial}{\partial v}$.

By calculation, $\xi$ has the following invariants: $u^{2}+$ $v^{2}, w, z$ and $x \cdot \xi(\operatorname{arctg}(v / u))=1$, so we take the transformation

$u_{1}=u^{2}+v^{2}, \quad v_{1}=\operatorname{arctg}(v / u), \quad w_{1}=w$,

$x_{1}=x, \quad z_{1}=z$,

such that (4.1) becomes

$\dot{u}_{1}=0, \quad \dot{w}_{1}=-\frac{1}{2} u_{1}, \quad \dot{x}_{1}=-z_{1}$,

$\dot{z}_{1}=x_{1}+\frac{1}{2} b u_{1}, \quad \dot{v}_{1}=w_{1}-b z_{1}$.

Obviously, the Jacobian determinant of $\varphi$ is equal to 1 , so here we do not need the second transformation in the proof of Theorem 7. In fact, the first equations in (4.2) are already independent of $v_{1}$ and divergenceless, and the exact solutions of (4.2) become

$$
\begin{aligned}
& u_{1}(t)=\rho_{0}^{2}, \quad w_{1}(t)=-\frac{1}{2} \rho_{0}^{2} t+w_{0}, \\
& x_{1}(t)=\left(x_{0}+b \rho_{0}^{2}\right) \cos t-z_{0} \sin t-\frac{1}{2} b \rho_{0}^{2}, \\
& z_{1}(t)=\left(x_{0}+b \rho_{0}^{2}\right) \sin t+z_{0} \cos t, \\
& v_{1}(t)=-\frac{1}{4} \rho_{0}^{2} t^{2}+w_{0} t+b\left(x_{0}+b \rho_{0}^{2}\right) \cos t \\
& \quad-b z_{0} \sin t+v_{0},
\end{aligned}
$$

where $\rho_{0}, z_{0}, w_{0}, v_{0}$ and $x_{0}$ are initial values.

\section{Acknowledgement}

We are very grateful to Professor C.R. Doering for suggestions for this Letter. The work is supported by the NSF of China.

\section{References}

[1] G.R.W. Quispel, Phys. Lett. A 206 (1995) 26. 
[2] Z.H. Xia, Ergodic Theory Dyn. Syst. 12 (1992) 621.

[3] J.E. Marsdem, A. Weinstein, Rep. Math. Phys. 51 (1974) 121.

[4] M.S. Janaki, G. Ghosh, J. Phys. A 20 (1987) 3679.

[5] I. Mezie, S. Wiggins, J. Nonlinear Sci. 4 (1994) 157.

[6] F.G. Gascon, Phys. Lett. A 225 (1996) 269.
[7] D.B. Huang, X.H. Zhao, Z.R. Liu. Lie symmetry group reduction for preserving $n$-form systems and applications, Sci. Sin. A (1997), to appear.

[8] P.J. Olver, Application of Lie Group to Differential Equations (Springer Verlag, New York, 1986).

[9] E.N. Lorenz, V. Krishnumurthy, J. Atmos. Sci. 44 (1987) 2940. 